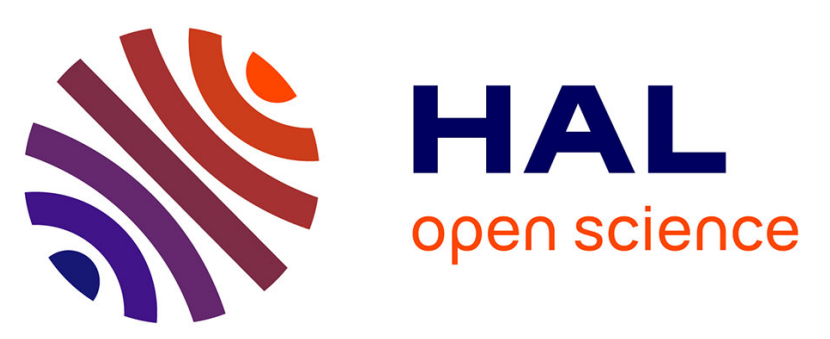

\title{
THE CONDUCTION-ELECTRON CONTRIBUTION TO THE ELECTRIC-FIELD GRADIENTS AT NUCLEAR SITES IN METALS
}

\author{
B. Kolk
}

\section{> To cite this version:}

B. Kolk. THE CONDUCTION-ELECTRON CONTRIBUTION TO THE ELECTRIC-FIELD GRADIENTS AT NUCLEAR SITES IN METALS. Journal de Physique Colloques, 1976, 37 (C6), pp.C6355-C6-358. 10.1051/jphyscol:1976667 . jpa-00216778

\section{HAL Id: jpa-00216778 https://hal.science/jpa-00216778}

Submitted on 1 Jan 1976

HAL is a multi-disciplinary open access archive for the deposit and dissemination of scientific research documents, whether they are published or not. The documents may come from teaching and research institutions in France or abroad, or from public or private research centers.
L'archive ouverte pluridisciplinaire HAL, est destinée au dépôt et à la diffusion de documents scientifiques de niveau recherche, publiés ou non, émanant des établissements d'enseignement et de recherche français ou étrangers, des laboratoires publics ou privés. 


\title{
THE CONDUCTION-ELECTRON CONTRIBUTION TO THE ELECTRIC-FIELD GRADIENTS AT NUCLEAR SITES IN METALS $\left({ }^{*}\right)$
}

\author{
B. KOLK $(* *)$ \\ Rutgers University, Department of Physics, Piscataway, New Jersey 08854, U. S. A.
}

\begin{abstract}
Résumé. - Il est démontré qu'il existe une forte corrélation entre la grandeur et le comportement en fonction de la température du gradient quadrupolaire électrostatique produit par les électrons de conductibilité à la position du noyau dans des échantillons métalliques, $e q_{c e}$, et la densité d'états électroniques au niveau de Fermi. En outre, divers mécanismes qui pourraient conđuire à la corrélation universelle entre $e q_{c e}$ et le gradient quadrupolaire de la maille sont décrits.
\end{abstract}

\begin{abstract}
It is shown that the magnitude and temperature behaviour of the conduction-electron contribution to the electric-field gradients at nuclear sites in metals, $e q_{\mathrm{ce}}$, are strongly correlated with the density of states at the Fermi surface. Furthermore, various mechanisms which may lead to the observed universal correlation between $e q_{\mathrm{cc}}$ and the lattice field gradient are studied.
\end{abstract}

1. Introduction. - The systematics of Electric-Field Gradients (EFG's) at nuclear sites in non-cubic metals is not very well understood. Traditionally this EFG is written as

$$
e q=\left(1-\gamma_{\infty}\right) e q_{\mathrm{lat}}+e q_{\mathrm{ce}}
$$

where $e q_{\text {lat }}$ is the ionic contribution due to the array of positive ions in a non-cubic lattice, $\gamma_{\infty}$ the Sternheimer factor which takes the shielding of $e q_{\text {lat }}$ by the core electrons into account, and $e q_{\mathrm{ce}}$ the EFG arising from the conduction electrons.

In many cases it is found that the total EFG, $e q$, has a sign opposite to $e q_{\text {lat }}$ and a temperature behavior which is also quite different from $e q_{\text {lat }}$. These differences between $e q$ and $e q_{\text {lat }}$ have been attributed to the presence of the EFG caused by the conduction electrons.

Recently, Raghavan, Kaufmann and Raghavan [1], determined $e q_{\mathrm{ce}}$ empirically by subtracting the ionic contribution, $\left(1-\gamma_{\infty}^{\prime}\right) e q_{1 a t}$, from measured EFG's, using Sternheimer factors, $\gamma_{\infty}^{\prime}$, calculated for free ions. The EFG, eqce, thus obtained plotted versus $\left(1-\gamma_{\infty}^{\prime}\right) e q_{12 t}$ led to the surprising correlation shown in figure 1 of ref. [1], which in crude approximation can be written as

$$
e q_{\mathrm{ce}}^{\prime} \cong-K\left(1-\gamma_{\infty}^{\prime}\right) e q_{\mathrm{lat}}
$$

with $K \simeq 2$.

Existing detailed calculations [2] of $e q_{\mathrm{ce}}$ do not predict such a correlation at all. In section 3 the

(*) Work supported by NSF Grant DMR76-02053.

(**) Present address : Department of Physics, Boston University, 111 Commington St., Boston, MA 02215, U. S. A. various mechanisms which may lead to such a correlation will be investigated.

First, however, we will consider (section 2) the conduction-electron field gradient without the perturbation of $e q_{\text {lat }}$, and study its temperature dependence and its relation to the density of states at the Fermi surface.

2. The conduction-electron field gradient. - The EFG at a nuclear site arising from the conduction electrons is given by

$$
e q_{\mathrm{ce}}=-\mathrm{e} \sum_{\mathbf{k}=0}^{\mathbf{k}_{\mathbf{F}}}\left\langle\psi_{\mathbf{k}}\left|2 \mathrm{P}_{2}(\hat{\mathbf{r}}) r^{-3}\right| \psi_{\mathbf{k}}\right\rangle
$$

where $\psi_{\mathbf{k}}(\mathbf{r})$ is the conduction-electron wavefunction. In this section the pure conduction-electron EFG $e q_{0}$, i. e. $e q_{\mathrm{ce}}$ without the perturbations arising from $e q_{\mathrm{lat}}$ (section 3), is investigated.

Recently, a study of the temperature dependence of $e q_{0}$ was reported by Jena $[3,4]$. Following conventional theory, Jena expressed $\psi_{\mathbf{k}}(\mathbf{r})$ in the pseudo-wave function $\varphi_{\mathbf{k}}(\mathbf{r})$, orthonormalized on the core-electron wavefunctions $\left|\alpha ; \mathbf{R}_{\mathrm{n}}\right\rangle$ of an atom at lattice site $\mathbf{R}_{\mathbf{n}}$;

$$
\begin{aligned}
& \psi_{\mathbf{k}}(\mathbf{r})= \\
= & C_{\mathbf{k}}\left[\varphi_{\mathbf{k}}(\mathbf{r})-\sum_{\mathbf{R}_{\mathbf{n}}} \mathrm{e}^{i \mathbf{k} \cdot \mathbf{R}_{n}} \sum_{\alpha}\left|\alpha ; \mathbf{R}_{\mathbf{n}}><\alpha\right| \varphi_{\mathbf{k}}>\right],
\end{aligned}
$$

where $C_{\mathrm{k}}$ is a normalization constant. Regarding the pseudo potential as a perturbation the pseudo wavefunction can be written as

$$
\varphi_{\mathbf{k}}(\mathbf{r})=\varphi_{\mathbf{k}}^{(0)}(\mathbf{r})+\varphi_{\mathbf{k}}^{(1)}(\mathbf{r})+\text { etc }
$$


which inserted in eq. (3) yields ;

$$
e q_{0}=e q_{0}^{(0)}+e q_{0}^{(1)}+\text { etc. . }
$$

The first and higher order terms in the expansion of $\varphi_{\mathbf{k}}(\mathbf{r})$ are temperature dependent;

$$
\varphi_{\mathbf{k}}^{(1)}(\mathbf{r}, T)=\sum_{\mathbf{g}}^{\prime} a_{\mathbf{g}} \mid \mathbf{k}+\mathbf{g}>\mathrm{e}^{-W(\mathbf{g}, T)}
$$

where $\mathbf{g}$ is a reciprocal lattice vector $W(\mathrm{~g}, T)$ the Debye-Waller factor which is related to the mean square displacement of a host atom, $\left\langle x_{\mathrm{T}}^{2}\right\rangle$, via $W(\mathrm{~g}, T)=g^{2}<x_{\mathrm{T}}>/ 2$. Using

$$
\exp \{-W(\mathrm{~g}, T)\} \cong 1-g^{2}<x_{\mathrm{T}}^{2}>/ 2
$$

one readily sees that the temperature dependence of $e q_{0}$ can be represented by

$$
e q_{0}=e q_{0}^{(0)}+e q_{0}^{(1)}\left(1-a<x_{\mathrm{T}}^{2}>\right) .
$$

The temperature dependence of $e q_{0}$ in tin [4], for example, is found to change by $40 \%$ with temperature, and it is not obvious from Jena's work that the temperature dependence in the first-order term of $e q_{0}$ is able to produce such a large temperature variation. Therefore $e q_{0}^{(0)}$ has to be scrutinized.

The zero-order term of $\varphi_{\mathbf{k}}(\mathbf{r})$ is a plane wave, which can be expanded in a series of spherical Bessel functions, $j_{l}(z)$, and spherical harmonics, $Y_{l m}(z)$ :

$$
\varphi_{\mathbf{k}}^{(0)}(\mathbf{r}) \sim \sum_{l, m} \boldsymbol{l}^{l} j_{l}(k r) Y_{l m}^{*}(\hat{k}) Y_{l m}(\hat{r})
$$

Inserting this series expansion in eq. (4) one readily sees that the zero-order conduction-electron wavefunction can formally be written as

$$
\psi_{\mathbf{k}}^{(0)}(\mathbf{r})=\sum_{l, m} \xi_{1}(k r) Y_{l m}^{*}(\mathbf{k}) Y_{l m}(\hat{r})
$$

which inserted in eq. (3) leads to

$$
\begin{aligned}
e q_{0}^{(0)}= & -2 \mathrm{e} \sum_{\substack{l, m \\
l^{\prime}=l, l \pm 2}}<Y_{l m}\left|2 \mathrm{P}_{2}\right| Y_{l^{\prime} m}>\times \\
& \times \sum_{\mathbf{k}=0}^{\mathbf{k}_{F}}<\xi_{l}\left|r^{-3}\right| \xi_{l^{\prime}}>Y_{l m}(\hat{k}) Y_{l^{\prime} m}(\hat{k}) .
\end{aligned}
$$

Consider the states with $|\mathbf{k}|<k_{0}$, where $k_{0}$ is the radius of the maximum sphere which can be inscribed within the Fermi surface. For these states one can sum or integrate over all directions of the wave vector $\mathbf{k}$, so that the $l^{\prime}=l \pm 2$ terms in eq. (8) will disappear, because of the orthogonality properties of $Y_{l m}(\hat{k})$. Since

$$
\sum_{m}<Y_{l m}\left|2 P_{2}\right| Y_{I m}>=0
$$

one readily sees that also the $l=l^{\prime}$ terms in eq. (8) yield a zero contribution for $|\mathbf{k}|<k_{0}$. Hence, only the occupied states outside the sphere with radius $k_{0}$ contribute to $e q_{0}^{(0)}$ so that in fair approximation :

$$
e q_{0}^{(0)}=-e \eta\left(E_{\mathrm{F}}\right) \sum_{l, l^{\prime}=l, l \pm 2}<\xi_{l}\left(E_{\mathrm{F}}\right)\left|r^{-3}\right| \xi_{l^{\prime}}\left(E_{\mathrm{F}}\right)>A_{l l^{\prime}}
$$

where $E_{\mathrm{F}}$ is the Fermi energy, $\eta(E)$ the density of states $F$ on the shape of the Fermi surface. In the case of a spherical Fermi surface, $k_{0}=k_{\mathrm{F}}$ and $A_{l l^{\prime}}=0$.

Hence, when the $A_{l l}$, or the density of states at the Fermi surface are small, $e q_{0}^{(0)}$ is negligible with respect to $e q_{0}^{(1)}$ to which all occupied states contribute, so that eq. (6) simplifies to

$$
e q_{0} \cong e q_{0}^{(1)}\left(1-a<x_{\mathrm{T}}^{2}>\right),
$$

and the temperature dependence in the first-order term in $\varphi_{\mathbf{k}}(\mathbf{r})$ has become the temperature dependence in zero order in $e q_{0}$.

For large values of $\eta\left(E_{\mathrm{F}}\right)$, however, $e q_{0}$ is no longer negligible, and eq. (9) indicates that in that cases a linear correlation between $e q_{0}$ and $\eta\left(E_{\mathrm{F}}\right)$ exists. In this case also a more complicated temperature behaviour of the EFG at a nuclear site as predicted by eq. (6) is expected, since electrons are excited to states above the Fermi level when temperature is increased. This changes the $l$ character of electrons in the vicinity of the Fermi level, and in turn changes the EFG at the nuclear site (see section 4 ).

3. The perturbation of $e q_{\text {ce }}$ by $e q_{1 a t}$. - In an attempt to explain the correlation observed by Raghavan et al. [1] the perturbation of $e q_{\mathrm{ce}}$ by the potential $V_{2}$,

$$
V_{2}(\mathbf{r})=-e r^{2} 2 \mathrm{P}_{2}(\hat{r}) e q_{\text {lat }}
$$

is studied next. In first-order perturbation theory $e q_{\text {ce }}$ can be expressed as

$$
e q_{\mathrm{ce}}=e q_{0}+e q_{1}+e q_{2}+e q_{3}
$$

where $e q_{0}$ is the pure conduction-electron EFG dealt with in section 2 and $e q_{1}, e q_{2}$, and $e q_{3}$ are associated with perturbation by $V_{2}$ of the conduction-electron energies, the conduction-electron wave-functions and the core-electron wavefunctions, respectively.

Due to the perturbation of the energy states by $V_{2}$ conduction electrons in the vicinity of the Fermi surface may occupy states with different $l$ character. Such a repopulation at the Fermi surface results in a change of the EFG and the nuclear site which is represented by $e q_{1}$. Watson, Gossard and Yafet [5] showed that $e q_{1}=-c \eta\left(E_{\mathrm{F}}\right) e q_{\text {lat }}$ and calculated values of $c \eta\left(E_{\mathrm{F}}\right)$ which are of the same order of magnitude as $K\left(1-\gamma_{\infty}\right)$. This result seems promising in order to explain eq. (2). However, the $e q_{1}$ calculated with the aid of eq. (12) in ref. [5] represents the EFG of conduction-electrons above the unperturbed Fermi surface and does not correct fot the fact that these electrons also produce an 
EFG in their unperturbed states which is of the same order of magnitude [6]. Hence, the actual value of $e q_{1}$ is expected to be at least one order of magnitude smaller than the values given by Watson et al. [5], so that $e q_{1}$ becomes too small to be responsible for a correlation such as given by eq. (2).

The $e q_{2}$ term arises from the perturbation of the conduction-electron wavefunction by $V_{2}$. According to Watson et al. [5] $e q_{2}$ is smaller than

$$
\left|\gamma_{n l}\right| e q_{\mathrm{lat}} z / 2(2 l+1)
$$

where $\gamma_{n, l}$ is the shielding factor of the $(n, l)$ valence shell in a neutral atom and $z$ is the number of conduction electrons per site. Hence, $e q_{2}$ is positively correlated to $e q_{\text {lat }}$ as opposed to the negative correlation empirically found (see Eq.(2)).

Besides the terms $e q_{1}$ and $e q_{2}$ already discussed by Watson $e t$ al. [5] we consider here a new term, $e q_{3}$, which arises from the fact that the conductionelectron wavefunctions are affected by the perturbation of those of the core electrons because they have to be orthogonal to each other. The core-electron wavefunctions are perturbed by the lattice EFG and by the quadrupole field of the nucleus, so that

$$
|\alpha\rangle=\left|\alpha^{(0)}\right\rangle+\left|\alpha^{\prime}\right\rangle \text {. }
$$

The perturbation $\left|\alpha^{\prime}\right\rangle$ gives rise to the shielding factor $\gamma_{\alpha}$;

$$
\left.-\gamma_{\alpha}=-2 e<\alpha^{(0)}\left|2 \mathbf{P}_{2}(\hat{\boldsymbol{r}}) r^{-3}\right| \alpha^{\prime}\right\rangle
$$

The Sternheimer factor is given by $\gamma_{\infty}=\sum_{\alpha} \gamma_{\alpha}$.

One readily sees from eq. (3) using eq. (13) that

$$
e q_{3}=-\gamma_{\beta} e q_{\mathrm{lat}} \sum_{\mathbf{k}} C_{\mathbf{k}}^{2}\langle\beta \mid \varphi\rangle\langle\varphi \mid \beta\rangle
$$

where $\beta$ denotes the $(n, l)$ quantum numbers of the most outer core shell. The contributions to $e q_{3}$ from the inner core shells are neglected. Furthermore, we have for sake of simplicity neglected the effect of $\left|\alpha^{\prime}\right\rangle$ on the orthogonalization coefficients $\langle\alpha \mid \varphi\rangle$. Since in good approximation $-\gamma_{\beta} \simeq-\gamma_{\infty} \simeq 1-\gamma_{\infty}$, $e q_{3}$ is proportional to $\left(1-\gamma_{\infty}\right) e q_{\text {lat }}$ in accordance with the empirical correlation, eq. (2). However, the correlation coefficient

$$
K=-\sum_{\mathbf{k}} C_{\mathbf{k}}^{2}\langle\beta \mid \varphi\rangle\langle\beta \mid \varphi\rangle
$$

is found to be of the order of -0.1 in disagreement with the value $K \cong+2$ obtained empirically.

If $e q_{3}$ is added to the lattice EFG, the Sternheimer factor if effectively increased by about $10 \%$.

4. Discussion. - It has been known [5] for some time that in certain cases a linear correlation between the EFG at the nuclear site and the density of states at the Fermi level, $\eta\left(E_{F}\right)$, exists, Watson, Gossard and Yafet [5] attributed this effect to the term $e q_{1}$ in eq. (12). In section 3 it was shown, however, that in ref. [5] $e q_{1}$ is overestimated, and thus does not dominate the other terms in eq. (12). In section 2 it was found that instead the pure conduction-electron contribution, $e q_{0}$, is strongly related to $\eta\left(E_{\mathrm{F}}\right)$.

Furthermore, we showed in section 2 that for small values of $\eta\left(E_{\mathrm{F}}\right)$ the temperature behaviour of $e q_{0}$ is given by eq. (10). To elucidate the physics which leads to this result, we describe the conduction electrons by simple plane waves, $e^{i k, r}=|\mathbf{k}\rangle$, which are scattered by the lattice atoms. The plane waves $|\mathbf{k}\rangle$ don't produce an EFG since $\left\langle\mathbf{k}\left|2 \mathrm{P}_{2} r^{-3}\right| \mathbf{k}\right\rangle=0$. The EFG at the impurity site arises from the mixing of the plane waves $\mid \mathbf{k}>$ with the Bragg reflected waves $\mid \mathbf{k}+\mathbf{g}>$ which yields an EFG at the nuclear site of $\left.-e<\mathbf{k}\left|2 \mathbf{P}_{2} r^{-3}\right| \mathbf{k}+\mathbf{g}\right\rangle$. As well known the thermal motion of the host atoms reduces the amplitude of the Bragg reflected waves. This effect leads to the temperature dependence given by eq. (10), where $\left\langle x_{\mathbf{T}}^{2}\right\rangle$ is thus the mean-square displacement of the host atoms. The consequence of this result is that the EFG at an impurity site should have the same temperature behaviour as that of the EFG at the host sites. This effect has indeed been observed for various impurities in a number of hosts [7].

The fact that the plane waves $|\mathbf{k}\rangle$ have to be orthogonal to the core wavefunctions yields the term $e q_{0}^{(0)}$ in eq. (6), which is linear in $\eta\left(E_{\mathrm{F}}\right)$ as shown by eq. (9). In the case where $\eta\left(E_{\mathrm{F}}\right)$ is large, $e q_{0}^{(0)}$ as well as $e q_{0}^{(1)}$ in eq. (6) will be affected by the change in occupation of the levels in the vicinity of the Fermi surface as function of temperature. The resulting increase in $p$ or $d$ character may increase $e q_{0}$ and in part compensate for the decrease in $e q_{0}$ arising from the temperature motion of the host atoms discussed above. When an impurity is introduced in the lattice, $\eta\left(E_{\mathrm{F}}\right)$ in the local surroundings of the impurity may change and thus affect the temperature behaviour of the EFG at the impurity nucleus, as has been observed for various impurities in $\mathrm{Ti}$ and Be hosts $[8,9]$.

A direct comparison of theory with experimental values of EFG's is very difficult as long as the origin of the empirical universal correlation found by Raghavan, Kaufmann, and Raghavan [1] is not understood. In order to explain this universal correlation the perturbation of the conduction-electron contribution to the EFG at a nuclear site, $e q_{\text {ce }}$, by the lattice EFG, $e q_{\text {lat }}$, was studied in section 3 . The terms $e q_{1}$ and $e q_{2}$ in eq. (12) are proportional to $e q_{1 a}$, and $e q_{3}$ is proportional to $\left(1-\gamma_{\infty}\right) e q_{\mathrm{lat}}$. However, none of these terms can be responsible for the empirically found correlation (eq. (2)) since either the sign of their correlation with $e q_{\text {lat }}$ is wrong or their order of magnitude is too small. 


\section{References}

[1] Raghavan, R. S., Kaufmann, E. N. and Raghavan, P., Phys. Rev. Let. 20 (1975) 1280.

[2] Das, T. P., Physica Scripta 11 (1975) 121.

[3] JENA, P., Phys. Rev. Let. 36 (1976) 418.

[4] Similar results as reported in ref. [3] were obtained by Collins from APW calculations of the temperature dependence of the EFG at nuclear sites in tin. Collins, G., Thesis Rutgers University (1976) unpublished.

[5] Watson, R. E., Gossard, A. C. and Yafet, Y., Phys. Rev. A 140 (1965) 375.

[6] In ref. [5] the shift of the Fermi level, $\delta E_{\mathrm{F}}$, required by the conservation of particles is taken as zero. Thus the number of conduction electrons is not conserved in the calculations given in ref. [5].

[7] Krien, K., Soares, J. C., Freitag, K., Vianden, R. and BIBILONI, A. G., Hyperfine Interactions 1 (1975) 217 ; Krien, K., Soares, J. C., Vianden, R., Bibiloni, A. G. and HANSEN, A., Hyperfine Interactions 1 (1975) 295.

[8] Reno, R. C., Raséra, R. L. and SchmidT, G., Phys. Lett. S0A (1974) 243.

[9] Krien, K., Soares, J. C., Hanser, A. and Feurer, B., Hyperfine Interactions, in press. 